\title{
McKeown or Ivor Lewis minimally invasive esophagectomy: a systematic review and meta-analysis
}

\author{
Jingpu Wang ${ }^{1}$, Jingfeng $\mathrm{Hu}^{1}$, Dengyan Zhu ${ }^{1}$, Kankan Wang ${ }^{2}$, Chunzhi Gao ${ }^{3}$, Tingting Shan ${ }^{1}$, Yang Yang ${ }^{1}$ \\ ${ }^{1}$ Department of Thoracic Surgery, ${ }^{2}$ Department of Nephrology, The First Affiliated Hospital, Zhengzhou University, Zhengzhou 450052, China; \\ ${ }^{3}$ Department of Spinal Orthopedics, General Hospital of Pingmei Shenma Medical Group, Pingdingshan 467000, China \\ Contributions: (I) Conception and design: J Wang; (II) Administrative support: Y Yang, D Zhu; (III) Provision of study materials or patients: J Wang, J \\ $\mathrm{Hu}$, T Shan; (IV) Collection and assembly of data: J Wang, J Hu; (V) Data analysis and interpretation: K Wang, C Gao; (VI) Manuscript writing: All \\ authors; (VII) Final approval of manuscript: All authors. \\ Correspondence to: Yang Yang, PhD. The First Affiliated Hospital of Zhengzhou University, Zhengzhou University, No.1 East Jianshe Road, \\ Zhengzhou 450052, China. Email: fccyangy1@zzu.edu.cn.
}

Background: Minimally invasive esophagectomy (MIE) is increasingly accepted in many countries. McKeown esophagectomy and Ivor Lewis esophagectomy are two protocols commonly used for MIE, but which one provides more benefit to the patients remains matter of controversy.

Methods: All records in PubMed, Embase, Medline, The Cochrane Library, Wanfang Database, China National Knowledge Infrastructure (CNKI) and Chinese VIP Information till May 2019 were systematically retrieved to compare the cohort studies of McKeown esophagectomy and Ivor Lewis esophagectomy. A meta-analysis of the extracted data was performed using the Review Manager 5.3 and Stata 15 software.

Results: The meta-analysis included 23 cohort studies in which a total of 4,933 patients were enrolled. The results revealed that minimally invasive McKeown esophagectomy (MIME) was superior to minimally invasive Ivor Lewis esophagectomy (MILE) in hospital cost, but inferior to it in operating time, length of hospital stay, in-hospital mortality, 30-day mortality, 90-day mortality, anastomotic leakage, anastomotic leakage requiring surgery, anastomotic stenosis, recurrent laryngeal nerve (RLN) injury, chylothorax, pulmonary complications and total complications. There were no statistical differences between MIME and MILE in blood loss, detected number of lymph nodes, blood transfusion rate, R0 resection rate, re-operation rate, drainage duration, length of the stay in intensive care unit (ICU), 1-year mortality, lung infection, cardiac arrhythmia and delayed gastric emptying.

Conclusions: Except for the cost, MILE is superior to MIME in several aspects, and may represent a better choice for MIE. The results of the present study should be interpreted with caution since the metaanalysis is based on nonrandom cohort studies which may have a selection bias.

Keywords: Minimally invasive esophagectomy (MIE); Ivor Lewis esophagectomy; McKeown esophagectomy; cervical anastomosis; intrathoracic anastomosis

Submitted Sep 26, 2019. Accepted for publication Jan 06, 2020.

doi: $10.21037 /$ tcr.2020.01.45

View this article at: http://dx.doi.org/10.21037/tcr.2020.01.45

\section{Introduction}

Esophageal cancer is one of the most common cancers worldwide, with the highest incidence in the middleand low-income countries (1). Esophageal cancer related mortality continues to increase (2), and in 2016 there were approximately 455,800 cases of this tumor and 400,200 deaths (1). Although considerable progress has been made in the diagnosis and treatment of esophageal cancer, globally, the overall 5 -year survival rate is only $15-20 \%$ (3). Currently, esophageal resection and systematic lymph node dissection regarded as the best treatment methods for this 
type of cancer (4).

With the development of minimally invasive techniques and related devices, a growing body of evidence indicates that minimally invasive esophagectomy (MIE) has clear advantages over open esophagectomy. It is being increasingly accepted in many countries and has become the standard method for esophageal resection (5). Transhiatal esophagectomy, McKeown esophagectomy and Ivor Lewis esophagectomy are the top three procedures for MIE (6). Minimally invasive McKeown esophagectomy (MIME) and minimally invasive Ivor Lewis esophagectomy (MILE) are becoming more frequently utilized by surgeons since they allow a more thorough dissection of the thoracic lymph node (7). However, whether MIME of MILE has more clinical advantages and should become the recommended procedure remains a matter of controversy (8).

To identify the differences in short-term outcomes between the two procedures, meta-analyses have been conducted using intraoperative data, primary postoperative complications, and short-term mortality. Despite the high value of this research and the inclusion of several Chinese studies, data available from Chinese databases were not utilized. This is an important omission since according to World Health Organization, there were 572,034 new cases of esophageal cancer worldwide in 2018 and 307,359 of them were in China, accounting for $53.73 \%$ of global incidence. Therefore, it is critical to include the Chinese database in the meta-analysis. Additionally, the secondary endpoints considered in the previous studies have their limitations and the differences between two surgical procedures were not analyzed in a comprehensive way.

This report presents a systematic review and metaanalyses of the outcomes of MIME and MILE, utilizing both Chinese and English-language databases. The inclusion of the Chinese database allowed collecting a greater abundance of research data, resulting in a more comprehensive evaluation of the differences between the two surgical procedures.

\section{Methods}

This meta-analysis adheres to the requirements of the Preferred Reporting Items for Systematic Reviews and Meta-Analyses (PRISMA) statement. The systematic review and meta-analysis have been registered in the PROSPERO database (registration number: CRD42019128884). An IRB approval and informed consent of the patients were not required for this systematic review.

\section{Literature search strategy}

All results pertinent to the use of MIME and MILE available from PubMed, Embase, Medline, The Cochrane Library, Wanfang Database, China National Knowledge Infrastructure (CNKI), and Chinese VIP Information, deposited on or before May 2019, were retrieved using the search strategies designed by the authors (Supplementary file 1). Only studies published in Chinese and English were included, regardless of the language of the country where the study was performed. To ensure that the retrieval is as comprehensive as possible, and to reduce the selection bias, all references listed in the included studies were retrieved and analyzed manually.

\section{Study selection}

The literature retrieval was carried out independently by two assessors (J Wang and $\mathrm{J} \mathrm{Hu}$ ) and the cohort studies that did not fulfill the inclusion criteria were screened out. First, studies that based on the title and the abstract were evidently unrelated to MIME or MILE were disregarded. Subsequently, the considered studies were downloaded in full-text format and assessed according to inclusion criteria. Inclusion criteria were as follows: (I) research type: cohort studies (including prospective cohort studies and retrospective cohort studies); (II) surgical procedures: clear description of the surgical procedures, ensuring that the surgical protocol complied with the criteria of minimally invasive surgery; and (II) research results: presence of the data on anastomotic leakage. Any disagreement during the retrieval and screening process was discussed with and resolved by the third assessor ( $Y$ Yang).

\section{Data extraction}

The data were extracted and exchanged for examination by two assessors (J Wang and $\mathrm{J} \mathrm{Hu}$ ) independently. Any disagreement was discussed with and resolved by the third assessor (Y Yang). The extracted information included: (I) paper information: name of the first author, publication year, duration of the study, study design, and country; (II) patient information: number of enrolled patients, age, gender, and tumor site; and (III) the outcome parameters. The primary outcome parameter was the occurrence of an 
anastomotic leakage, while secondary outcome parameters included operating time, blood loss, number of lymph nodes obtained, R0 resection rate, drainage duration, re-operation rate, blood transfusion rate, length of stay in intensive care unit (ICU), length of hospital stay, cost, in-hospital mortality, 30-day mortality, 90-day mortality, 1-year mortality, anastomotic stenosis, recurrent laryngeal nerve injury, chylothorax, pulmonary complications, arrhythmia, delayed gastric emptying and total complications.

\section{Quality assessment}

Quality assessment was performed using the NewcastleOttawa quality assessment scale (NOS). The scale comprises 8 items divided into three parts: selection (maximum score, 4), comparability (maximum score, 2), and outcome (maximum score, 3 ) and which in total can give a maximum score of 9 points. The total score of 7 or more points was considered to indicate a high quality of the study, 6 points medium quality, and less than 6 points indicate low quality. Only studies with high and medium quality were finally enrolled. The assessment process was carried out independently by two assessors (J Wang and J Hu). Any disagreement was discussed with the third assessor until an agreement was reached.

\section{Statistical analysis}

Statistical analysis of the data was conducted using the Review Manager 5.3 software. The heterogeneity between the studies was analyzed by the $\mathrm{I}^{2}$ test, and $\mathrm{I}^{2}>50 \%$ was considered to indicate significant heterogeneity between the studies. When significant heterogeneity was present between the studies, DerSimonian and Laird random effects model or Mantel-Haenszel fixed effect model was chosen. For binary variable results, the odds ratio (OR) and $95 \%$ confidence interval (CI) were calculated. For continuous variables, the mean difference and $95 \%$ CI were computed. $\mathrm{P}<0.05$ was considered as statistically significant. Sensitivity analysis was performed on variables with significant heterogeneity using the Stata 15 statistical package to identify the source of heterogeneity. The publication bias of variables with the number of studies $>10$ was tested using the Egger's test. The trim-and-fill method was used to further test the influence of publication bias on the results.

\section{Results}

\section{Literature search and quality assessment}

The retrieval and screening process of the references is shown in Figure 1. The retrieval strategy used identified 5,728 studies, of which 4,108 remained after removing duplicate publications. After reading the titles and abstracts, 4,028 studies that were evidently unrelated to the present analysis were eliminated. Full text of the remaining 80 studies was downloaded for further detailed reviews. Finally, the meta-analysis was conducted on the 23 studies that met the inclusion criteria. The characteristics and quality assessment scores of these 23 investigations are listed in Table S1. The quality assessment scores for all studies were above 6, meeting the inclusion criteria (8-30).

\section{Results of meta-analysis}

\section{Primary outcome measures Anastomotic leakage}

A total of 23 publications reported the occurrence rate of anastomotic leakage. However, the report by Luketich et al. (21) provided only the incidence of anastomotic leakage requiring surgery, making it impossible to combine it with the other studies. This necessitated a separate metaanalysis of the occurrence rate of anastomotic leakage requiring surgery (see below). The 22 studies which listed the incidence of anastomotic leakage included 3,922 patients. The occurrence rate of anastomotic leakage was $14.7 \%$ in MIME, and $5.5 \%$ in MILE. The heterogeneity between the 22 studies was not statistically significant $\left(\mathrm{P}=0.09 ; \mathrm{I}^{2}=30 \%\right)$. Therefore, the fixed-effect model was adopted to compare the outcomes of the two protocols. This analysis indicated that the occurrence rate of anastomotic leakage was higher in MIME than that in MILE (OR =2.97, 95\% CI: 2.34-3.77), and this difference was statistically significant $(\mathrm{P}<0.00001)$ (Figure 2$)$.

Three studies, reporting on 1,541 patients, included the data on the incidence of anastomotic leakage requiring surgery. The heterogeneity between the studies was not statistically significant $\left(\mathrm{P}=0.51 ; \mathrm{I}^{2}=0 \%\right)$; therefore, the fixed-effect model was chosen for analysis, which indicated 


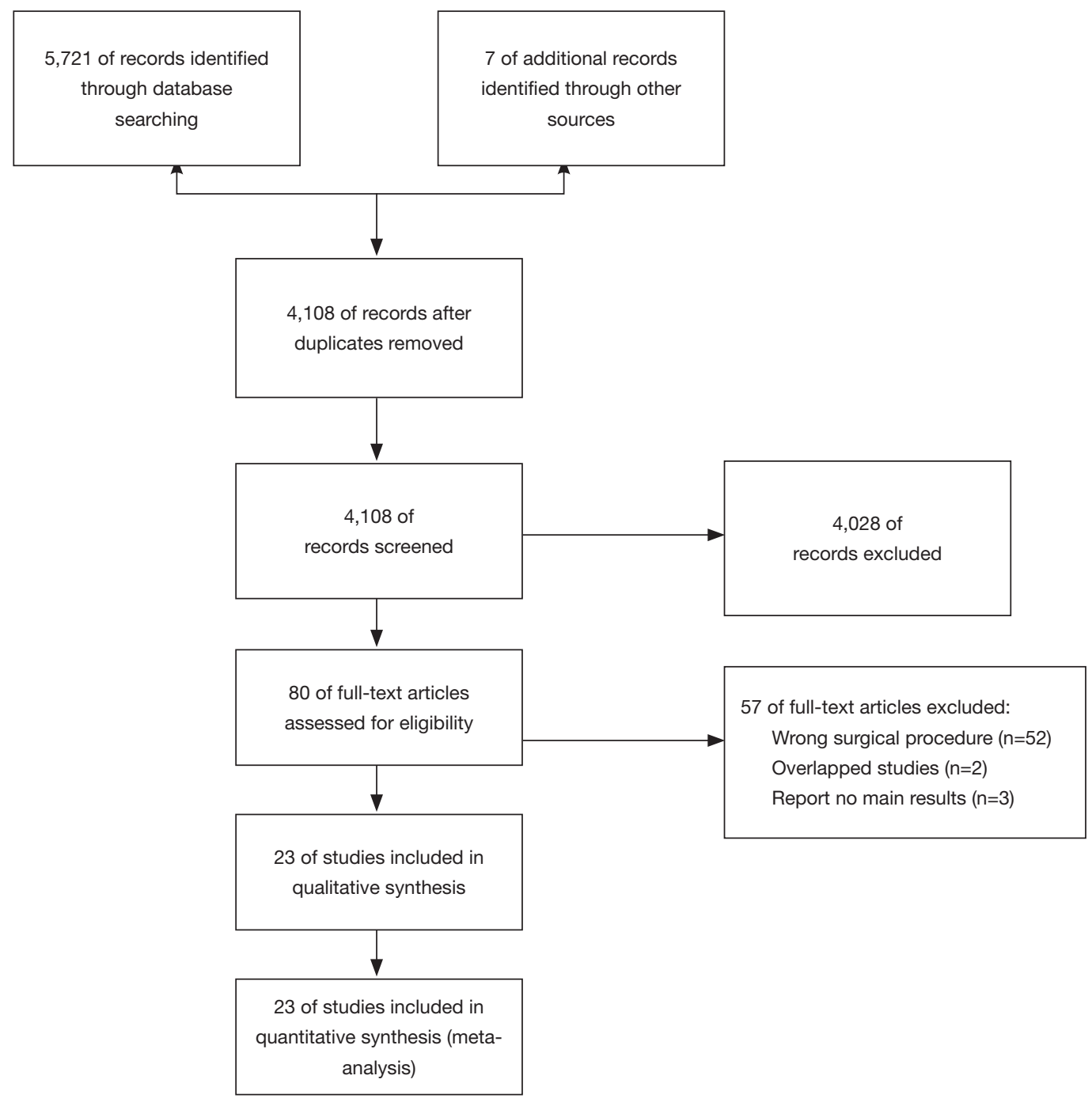

Figure 1 Summary of screening and selection process.

that the occurrence rate of anastomotic leakage requiring surgery was significantly higher in MIME than in MILE $(\mathrm{OR}=1.57,95 \%$ CI: 1.02-2.43, $\mathrm{P}=0.04)$ (Figure 3).

\section{Secondary outcome measures Intraoperative data}

The results of the meta-analysis of intraoperative data are shown in Table 1. The operating time was reported in 20 studies that included 3,478 patients. Meta-analysis documented that the operating time of MIME was longer than that of MILE [weighted mean difference (WMD) $=23.69$, 95\% CI: 6.26-41.12, P=0.008]. There was no statistically significant difference between the two surgical procedures in terms of blood loss, the number of lymph nodes detected, transfusion rate, and $\mathrm{R} 0$ resection rate.

\section{Postoperative data}

The results of the meta-analysis of postoperative data are listed in Table 2. In comparison with MILE, MIME was associated with a longer length of hospital stay (WMD $=1.13,95 \%$ CI: $0.45-1.82, \mathrm{P}=0.001)$, higher in-hospital mortality ( $\mathrm{OR}=2.83,95 \% \mathrm{CI}: 1.35-5.93, \mathrm{P}=0.006)$, higher 30-day mortality $(\mathrm{OR}=2.44,95 \% \mathrm{CI}: 1.33-4.50, \mathrm{P}=0.004)$, higher 90-day mortality (OR $=2.85,95 \% \mathrm{CI}: 1.55-5.23$, $\mathrm{P}=0.0007)$, but the cost of MIME was lower (WMD $=-0.40$, $95 \% \mathrm{CI}:-0.74$ to $0.07, \mathrm{P}=0.02)$. The re-operation rate, drainage duration, length of stay in the ICU, and 1-year mortality were not statistically different between the two surgical procedures. 


\begin{tabular}{|c|c|c|c|c|c|c|c|c|c|c|}
\hline Study or Subgroup & $\begin{array}{l}\text { McKeo } \\
\text { Events }\end{array}$ & $\begin{array}{l}\text { wn } \\
\text { Total }\end{array}$ & $\begin{array}{l}\text { Nor Le } \\
\text { Events }\end{array}$ & $\begin{array}{l}\text { wis } \\
\text { Total }\end{array}$ & Weight & $\begin{array}{c}\text { Odds Ratio } \\
\text { M-H, Fixed, } 95 \% \mathrm{Cl}\end{array}$ & & $\begin{array}{r}\text { Odds F } \\
\text { M-H, Fixed }\end{array}$ & $\begin{array}{l}\text { Ratio } \\
\text { d, } 95 \% \mathrm{Cl}\end{array}$ & \\
\hline Brown et al 2017 & 4 & 61 & 1 & 49 & $1.2 \%$ & $3.37[0.36,31.16]$ & & & & \\
\hline Chang 2018 & 4 & 33 & 4 & 20 & $5.1 \%$ & $0.55[0.12,2.51]$ & & & & \\
\hline Chen et al 2017 & 4 & 51 & 1 & 200 & $0.4 \%$ & $16.94[1.85,155.04]$ & & & & \\
\hline Gao 2016 & 9 & 77 & 2 & 81 & $2.0 \%$ & $5.23[1.09,25.03]$ & & & & \\
\hline Hao et al 2014 & 4 & 81 & 0 & 55 & $0.6 \%$ & $6.45[0.34,122.16]$ & & & & \\
\hline Hou et al 2017 & 8 & 65 & 3 & 120 & $2.1 \%$ & $5.47[1.40,21.42]$ & & & & \\
\hline Huang 2017 & 15 & 100 & 6 & 100 & $5.9 \%$ & $2.76[1.03,7.45]$ & & & & \\
\hline Lin et al 2014 & 24 & 185 & 2 & 103 & $2.6 \%$ & $7.53[1.74,32.54]$ & & & & \\
\hline Ling 2018 & 9 & 56 & 2 & 73 & $1.7 \%$ & $6.80[1.41,32.87]$ & & & & \\
\hline Mei 2016 & 12 & 65 & 2 & 41 & $2.3 \%$ & $4.42[0.93,20.86]$ & & & & \\
\hline Meng 2016 & 7 & 26 & 0 & 21 & $0.5 \%$ & $16.54[0.89,308.98]$ & & & & \\
\hline Nguyen et al 2008 & 3 & 47 & 4 & 51 & $4.2 \%$ & $0.80[0.17,3.78]$ & & & & \\
\hline Ren 2018 & 6 & 41 & 1 & 41 & $1.0 \%$ & $6.86[0.79,59.76]$ & & & & \\
\hline Rong et al 2018 & 10 & 65 & 2 & 65 & $2.0 \%$ & $5.73[1.20,27.27]$ & & & & \\
\hline Schmidt et al 2017 & 25 & 146 & 28 & 188 & $23.5 \%$ & $1.18[0.66,2.13]$ & & & - & \\
\hline Sun 2017 & 5 & 42 & 1 & 50 & $0.9 \%$ & $6.62[0.74,59.11]$ & & & & \\
\hline Tang et al 2016 & 30 & 278 & 4 & 155 & $5.3 \%$ & $4.57[1.58,13.22]$ & & & - & \\
\hline Wei et al 2016 & 6 & 50 & 0 & 70 & $0.4 \%$ & $20.60[1.13,374.61]$ & & & & \\
\hline Workum et al 2018 & 59 & 210 & 29 & 210 & $24.1 \%$ & $2.44[1.49,4.00]$ & & & $\rightarrow-$ & \\
\hline Wu et al 2014 & 20 & 138 & 13 & 219 & $9.9 \%$ & $2.69[1.29,5.60]$ & & & $\longrightarrow$ & \\
\hline Zhai 2016 & 12 & 62 & 3 & 50 & $3.1 \%$ & $3.76[1.00,14.16]$ & & & & \\
\hline Zhang et al 2018 & 7 & 49 & 1 & 32 & $1.2 \%$ & $5.17[0.60,44.18]$ & & & & \\
\hline Total $(95 \% \mathrm{Cl})$ & & 1928 & & 1994 & $100.0 \%$ & $2.97[2.34,3.77]$ & & & $\boldsymbol{\gamma}$ & \\
\hline Total events & 283 & & 109 & & & & & & & \\
\hline $\begin{array}{l}\text { Heterogeneity: } \mathrm{Chi}^{2} \\
\text { Test for overall effec }\end{array}$ & $\begin{array}{l}30.21, d f= \\
Z=8.97\end{array}$ & $\begin{array}{l}=21(P \\
(P<0.0\end{array}$ & $=0.09)$ & $I^{2}=309$ & & & 0.002 & $\begin{array}{l}0.1 \\
\text { [Mckeown] }\end{array}$ & $\begin{array}{c}10 \\
\text { Favours [lvo }\end{array}$ & 500 \\
\hline
\end{tabular}

Figure 2 Forest plot anastomotic leakage. CI, confidence interval; M-H, Mantel-Haenszel.

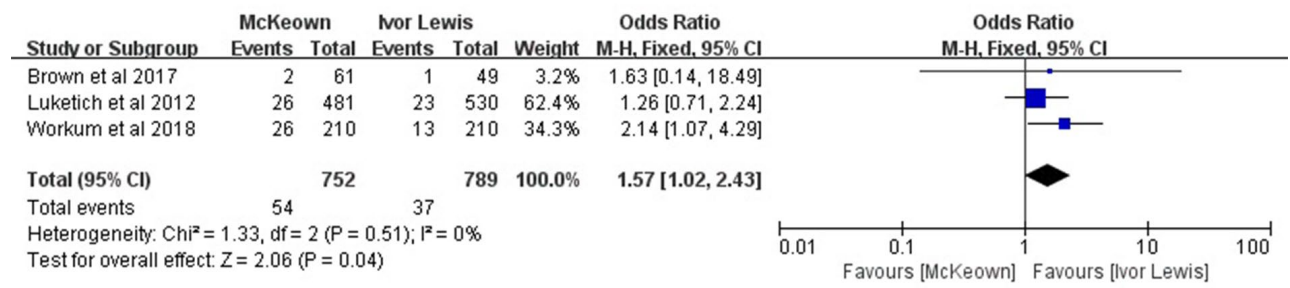

Figure 3 Forest plot anastomotic leakage requiring surgery. CI, confidence interval; M-H, Mantel-Haenszel.

\section{Complications}

The results of the meta-analysis of complications are presented in Table 3. Patients undergoing MIME had a higher morbidity associated with anastomotic stricture $(\mathrm{OR}$ $=2.89$, 95\% CI: 1.97-4.24, $\mathrm{P}<0.00001)$, recurrent laryngeal nerve $(\mathrm{RLN})$ injury $(\mathrm{OR}=5.63,95 \% \mathrm{CI}: 3.99-7.94$, $\mathrm{P}<0.00001)$, chylothorax (OR $=1.55$, 95\% CI: 1.01-2.38, $\mathrm{P}=0.04)$, pulmonary complications $(\mathrm{OR}=1.89$, 95\% CI: 1.54-2.32, $\mathrm{P}<0.00001)$ and overall complications $(\mathrm{OR}=1.90$, 95\% CI: $1.34-2.71, \mathrm{P}=0.0004)$. There were no statistical differences in morbidity related to lung infection, cardiac arrhythmia and delayed gastric emptying between MIME and MILE.

\section{Sensitivity analysis}

Evident heterogeneity was present in the nine groups of studied variables, including operating time $\left(\mathrm{I}^{2}=98 \%\right.$, $\mathrm{P}<0.00001)$, blood loss $\left(\mathrm{I}^{2}=71 \%, \mathrm{P}<0.00001\right)$, number of detected lymph nodes $\left(\mathrm{I}^{2}=87 \%, \mathrm{P}<0.00001\right)$, drainage duration $\left(\mathrm{I}^{2}=94 \%, \mathrm{P}<0.00001\right)$, length of hospital stay $\left(\mathrm{I}^{2}=82 \%, \mathrm{P}<0.00001\right)$, length of the stay in $\mathrm{ICU}\left(\mathrm{I}^{2}=95 \%\right.$, $\mathrm{P}<0.00001)$, cost $\left(\mathrm{I}^{2}=87 \%, \mathrm{P}<0.00001\right)$, lung infection $\left(\mathrm{I}^{2}=52 \%, \mathrm{P}=0.04\right)$, and total complications $\left(\mathrm{I}^{2}=74 \%\right.$, $\mathrm{P}<0.00001)$. The sensitivity analysis for blood loss, drainage duration, length of hospital stay, length of the stay in ICU, cost and lung infection produced stable results, indicating that there was no source of heterogeneity. The sensitivity analysis of operating time showed that studies by Tang et al. (16) and van Workum et al. (18) significantly influenced the combined OR and CI. However, when these studies were removed, there was no evident change in heterogeneity $\left(\mathrm{I}^{2}=98 \%, \mathrm{P}<0.00001\right)$. The sensitivity analysis of the number of detected lymph nodes suggested 
Table 1 Meta-analysis for intraoperative data

\begin{tabular}{|c|c|c|c|c|c|c|c|c|}
\hline Outcomes & $\begin{array}{l}\text { No. of } \\
\text { studies }\end{array}$ & $\begin{array}{c}\text { No. of } \\
\text { patients }\end{array}$ & $\begin{array}{l}\text { McKeown's } \\
\text { events } \\
\text { incidence (\%) }\end{array}$ & $\begin{array}{l}\text { Ivor Lewis's } \\
\text { events } \\
\text { incidence } \\
(\%)\end{array}$ & WMD/OR (95\% Cl) & Heterogeneity & Test for overall effect & $\begin{array}{l}\text { Favors } \\
\text { group }\end{array}$ \\
\hline $\begin{array}{l}\text { Operating } \\
\text { time }\end{array}$ & 20 & 3,478 & - & - & $23.69(6.26,41.12)$ & $I^{2}=98 \%, P<0.00001$ & $Z=2.66, P=0.008$ & $\begin{array}{l}\text { Ivor } \\
\text { Lewis }\end{array}$ \\
\hline Blood loss & 18 & 3,005 & - & - & $7.73(-2.21,17.59)$ & $\mathrm{I}^{2}=71 \%, \mathrm{P}<0.00001$ & $Z=1.54, P=0.12$ & - \\
\hline R0 resection & 5 & 2,095 & $97.2 \%$ & $96.8 \%$ & $1.07(0.63,1.80)$ & $\mathrm{I}^{2}=0 \%, P=0.67$ & $Z=0.24, P=0.81$ & - \\
\hline $\begin{array}{l}\text { Conversion } \\
\text { to open }\end{array}$ & 3 & 1,616 & $4.5 \%$ & $3.6 \%$ & $1.32(0.80,2.18)$ & $\mathrm{I}^{2}=0 \%, P=0.92$ & $Z=1.08, P=0.28$ & - \\
\hline
\end{tabular}

WMD, weighted mean difference; OR, odds ratio; Cl, confidence interval.

Table 2 Meta-analysis for postoperative data

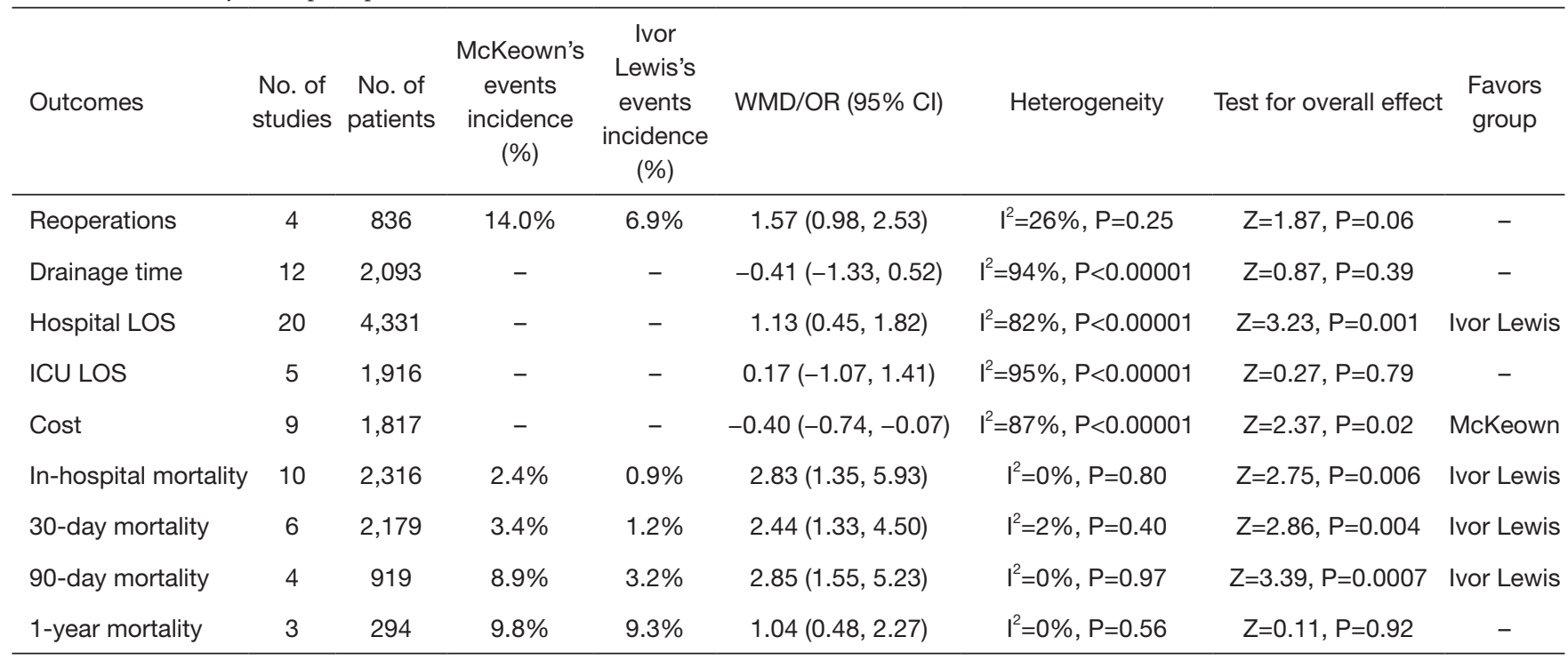

WMD, weighted mean difference; OR, odds ratio; Cl, confidence interval; LOS, length of stay.

that the study by Luketich et al. (21) significantly affected the final result. After eliminating this study, significant heterogeneity continued to be present $\left(\mathrm{I}^{2}=75 \%, \mathrm{P}<0.00001\right)$. Sensitivity analysis pointed to the publication of Tang et $a l$. as the source of heterogeneity in meta-analysis of total complications. After removing this study, the heterogeneity of the results disappeared $\left(\mathrm{I}^{2}=0 \%, \mathrm{P}<0.66\right)$, and the results were: $\mathrm{OR}=1.55,95 \%$ CI: $1.30-1.85, \mathrm{Z}=4.83, \mathrm{P}<0.00001$.

\section{Publication bias}

The Egger's test revealed that there was publication bias affecting the results of operating time, length of hospital stay, anastomotic stenosis, and anastomotic leakage. 
Table 3 Meta-analysis for complications data

\begin{tabular}{|c|c|c|c|c|c|c|c|c|}
\hline Outcomes & $\begin{array}{l}\text { No. of } \\
\text { studies }\end{array}$ & $\begin{array}{c}\text { No. of } \\
\text { patients }\end{array}$ & $\begin{array}{c}\text { McKeown's } \\
\text { events } \\
\text { incidence } \\
(\%)\end{array}$ & $\begin{array}{l}\text { Ivor } \\
\text { Lewis's } \\
\text { events } \\
\text { incidence } \\
\text { (\%) }\end{array}$ & WMD/OR $(95 \% \mathrm{Cl})$ & Heterogeneity & Test for overall effect & Favors group \\
\hline $\begin{array}{l}\text { Anastomotic } \\
\text { stricture }\end{array}$ & 11 & 2,102 & $10.0 \%$ & $4.1 \%$ & $2.89(1.97,4.24)$ & $\mathrm{I}^{2}=25 \%, P=0.21$ & $Z=5.43, P<0.00001$ & Ivor Lewis \\
\hline Chylothorax & 12 & 2,352 & $4.9 \%$ & $3.1 \%$ & $1.55(1.01,2.38)$ & $\mathrm{I}^{2}=0 \%, \mathrm{P}=0.70$ & $Z=2.03, P=0.04$ & Ivor Lewis \\
\hline $\begin{array}{l}\text { Pulmonary } \\
\text { complications }\end{array}$ & 14 & 2,736 & $25.4 \%$ & $15.1 \%$ & $1.89(1.54,2.32)$ & $\mathrm{I}^{2}=36 \%, P=0.09$ & $Z=6.11, P<0.00001$ & Ivor Lewis \\
\hline $\begin{array}{l}\text { Cardiac } \\
\text { arrhythmia }\end{array}$ & 10 & 1,789 & $6.0 \%$ & $4.3 \%$ & $1.39(0.89,2.18)$ & $\mathrm{I}^{2}=0 \%, \mathrm{P}=0.69$ & $Z=1.44, P=0.15$ & - \\
\hline $\begin{array}{l}\text { Delayed gastric } \\
\text { emptying }\end{array}$ & 9 & 1,537 & $4.8 \%$ & $3.9 \%$ & $1.33(0.80,2.23)$ & $\mathrm{I}^{2}=36 \%, P=0.13$ & $Z=1.09, P=0.28$ & - \\
\hline $\begin{array}{l}\text { Overall } \\
\text { complications }\end{array}$ & 13 & 2,861 & $43.8 \%$ & $29.7 \%$ & $1.90(1.34,2.71)$ & $\begin{array}{l}\mathrm{I}^{2}=74 \%, \mathrm{P} \\
<0.00001\end{array}$ & $Z=3.57, P=0.0004$ & Ivor Lewis \\
\hline
\end{tabular}

WMD, weighted mean difference; OR, odds ratio; $\mathrm{Cl}$, confidence interval; $\mathrm{RLN}$, recurrent laryngeal nerve.

However, the trim-and-fill analysis indicated that the publication bias did not affect significantly the conclusions.

\section{Discussion}

This systematic review and meta-analysis compare the short-term effects of MIME and MILE. The present study reveals for the first time that MILE is superior to MIME in in-hospital mortality, 30-day mortality, 90-day mortality, the incidence of chylothorax, severe anastomotic leakage, and total complications, but is associated with a higher cost. The analysis confirmed previous findings that MILE provides better outcomes of operating time, length of hospital stay, anastomotic leakage, anastomotic stenosis, RLN injury, pulmonary complications.

In the comparison of intra-operative data, only identified difference between the two surgical procedures was the shorter operating time in MILE, since the neck incision is not necessary with this protocol. Among the postoperative complications, the lifted length of the tubular stomach was shorter in MILE, reducing the tension of anastomotic stoma and providing a better blood supply. Additionally, the anastomotic stoma inside the thorax was less compressed than the anastomotic stoma in the narrow cervical region, decreasing the likelihood of anastomotic leakage and anastomotic stenosis. Since the neck incision was not required for MILE, the exposure of the recurrent cervical laryngeal nerve was avoided, decreasing the morbidity associated with RLN injury. The morbidity of pulmonary complications after MILE was also lower, likely due to the shorter operating and RLN injury $(31,32)$. MILE dissociated the proximal esophagus, which was lower than that in MIME, so it had less chance to damage the thoracic duct and reduced the morbidity of chylothorax (10). The meta-analysis of postoperative data indicated that MILE had lower morbidity associated with postoperative complications, resulting in a shorter length of hospital stay, and lower in-hospital mortality, 30-day mortality, and 90-day mortality. In MILE, anastomat was used for intrathoracic esophageal anastomosis, which may increase 
the cost (12). Importantly, all the relevant data were from Chinese research.

A remarkable aspect of the present analysis is the demonstration of the survival benefit of MILE. Mortality is an important indicator of the outcome of the surgery mode, highlighting the significance of the comparison of MILE and MIME. The absence of a statistically significant difference in 1-year mortality may reflect the small number of enrolled studies and cases was.

In comparison with previously published meta-analyses, more perfect improved strategies were developed, which not only enhanced the effectiveness of the search, but also retrieved the Chinese database. As a result, 23 medium and high-quality studies with 4,933 patients were enrolled in the meta-analysis. More outcome indexes were collected and analyzed, and the differences between the two procedures were analyzed in a more comprehensive way. The current meta-analysis detected also more differences between the two surgical approaches than previously published studies. Of note, a sensitivity analysis was performed for the results with significant heterogeneity, and the sources of heterogeneity were identified.

Some limitations of the present study should be acknowledged: (I) The analysis included only cohort studies so that the selection bias was unavoidable. (II) Long-term survival data were not available in the enrolled studies, making the assessment of the difference in long-term prognosis between MILE and MIME impossible. This limitation emphasized the need for new relevant clinical studies to determine the long-term survival after these two surgical protocols. (III) The homogeneity test of continuous variables demonstrated a significant heterogeneity. It may be caused by differences in experience and skills among the surgeons, and variations in the diagnosis and treatment protocols between different geographic regions. (IV) Among the 23 studies enrolled in the research, 18 were performed in China. Thus, the results of the present study may not serve as a reference for other countries and regions of the world.

\section{Conclusions}

MILE is superior to MIME in operating time, length of hospital stay, in-hospital mortality, 30-day mortality, 90day mortality, anastomotic leakage, anastomotic leakage requiring surgery, anastomotic stenosis, RLN injury, chylothorax, pulmonary complications, total complications. However, MILE is associated with a higher cost. Therefore,
MILE may represent a better option for MIE if economic conditions are not a limiting factor. It is worth noting that presented the meta-analysis is based on nonrandom cohort studies that have inherent selection bias and, therefore, should be interpreted cautiously. A large number of highquality randomized controlled trials should be done to verify the results.

\section{Acknowledgments}

We would like to thank Guangqi Qin, PhD and Jiao Liu, $\mathrm{PhD}$, for their help with correcting the manuscript and providing valuable support and comments during our scientific research.

Funding: None.

\section{Footnote}

Conflicts of Interest: All authors have completed the ICMJE uniform disclosure form (available at http://dx.doi. org/10.21037/tcr.2020.01.45). The authors have no conflicts of interest to declare.

Ethical Statement: The authors are accountable for all aspects of the work in ensuring that questions related to the accuracy or integrity of any part of the work are appropriately investigated and resolved.

Open Access Statement: This is an Open Access article distributed in accordance with the Creative Commons Attribution-NonCommercial-NoDerivs 4.0 International License (CC BY-NC-ND 4.0), which permits the noncommercial replication and distribution of the article with the strict proviso that no changes or edits are made and the original work is properly cited (including links to both the formal publication through the relevant DOI and the license). See: https://creativecommons.org/licenses/by-nc-nd/4.0/.

\section{References}

1. Torre LA, Siegel RL, Ward EM, et al. Global Cancer Incidence and Mortality Rates and Trends--An Update. Cancer Epidemiol Biomarkers Prev 2016;25:16-27.

2. Takahashi C, Shridhar R, Huston J, et al. Esophagectomy from then to now. J Gastrointest Oncol 2018;9:903-9.

3. Lagergren J, Smyth E, Cunningham D, et al. Oesophageal cancer. Lancet 2017;390:2383-96.

4. Rustgi AK, El-Serag HB. Esophageal carcinoma. N Engl J 
Med 2014;371:2499-509.

5. Gisbertz SS, Hagens ERC, Ruurda JP, et al. The evolution of surgical approach for esophageal cancer. Ann N Y Acad Sci 2018;1434:149-55.

6. Deng J, Su Q, Ren Z, et al. Comparison of short-term outcomes between minimally invasive McKeown and Ivor Lewis esophagectomy for esophageal or junctional cancer: a systematic review and meta-analysis. Onco Targets Ther 2018;11:6057-69.

7. van Workum F, Berkelmans GH, Klarenbeek BR, et al. McKeown or Ivor Lewis totally minimally invasive esophagectomy for cancer of the esophagus and gastroesophageal junction: systematic review and metaanalysis. J Thorac Dis 2017;9:S826-33.

8. Chen L, Liu X, Wang R, et al. Minimally invasive esophagectomy for esophageal cancer according to the location of the tumor: Experience of 251 patients. Ann Med Surg (Lond) 2017;17:54-60.

9. Huang S. Minimally invasive Ivor-Lewis Vs McKeown esophagectomy in the treatment of lower thoracic esophageal cancer. J MED Theor Prac 2017;30:3189-91.

10. Lin J, Kang M, Lin J, et al. Short-term efficacy comparison between Ivor-Lewis approach and McKeown approach in minimally invasive esophagectomy. Zhonghua Wei Chang Wai Ke Za Zhi 2014;17:888-91.

11. Meng Q. Clinical Analysis of Minimal Invasive McKeown and Ivor-Lewis Surgery in the Treatment of MiddleLower Section Esophageal Cancer. Medical Innovation of China 2016;13:17-20.

12. Wu H, Xie M, Liu C, et al. A retrospective study of short-term outcomes of minimally invasive Ivor-Lewis esophagectomy and McKeown esophagectomy for thoracic middle-lower esophageal carcinoma. Chin J Thorac Cardiovasc Surg 2014;30:649-52.

13. Hou X, Ren Z, Yang B, et al. Comparison of short-term therapeutic efficacy between minimally invasive IvorLewis esophagectomy and Mckeown esophagectomy for esophageal cancer. Biomed Res 2017;28:5321-6.

14. Rong B. Comparison of Ivor-Lewis approach and Mckeown approach in treating mid-thoracic esophageal cancer. Chin J Clin Healthc 2018;21:497-500

15. Wei H. The comparison of the clinical curative effect of Ivor-Lewis and McKeown operation in totally endoscopic esophagectomy. Chin J Thorac Cardiovasc Surg 2016;32:495-8.

16. Tang X. Short-term effects of full-endoscopic Ivorlews and Mckeown operations on treatment of thoracic middle-lower segment esophageal carcinoma. Medical
Journal of National Defending Forces in Southwest China 2016;26:1173-5.

17. Brown AM, Pucci MJ, Berger AC, et al. A standardized comparison of peri-operative complications after minimally invasive esophagectomy: Ivor Lewis versus McKeown. Surg Endosc 2018;32:204-11.

18. van Workum F, Slaman AE, van Berge Henegouwen MI, et al. Propensity Score-Matched Analysis Comparing Minimally Invasive Ivor Lewis Versus Minimally Invasive Mckeown Esophagectomy. Ann Surg 2020;271:128-33.

19. Sun Y. Feasibility, safety and short-term efficacy of thoracoscopy and laparoscopy combined with Ivor-Lewis surgery for middle and lower esophageal carcinoma. Hainan Med J 2017;28:576-7.

20. Nguyen NT, Hinojosa MW, Smith BR, et al. Minimally invasive esophagectomy: lessons learned from 104 operations. Ann Surg 2008;248:1081-91.

21. Luketich JD, Pennathur A, Awais O, et al. Outcomes after minimally invasive esophagectomy: review of over 1000 patients. Ann Surg 2012;256:95-103.

22. Schmidt HM, Gisbertz SS, Moons J, et al. Defining Benchmarks for Transthoracic Esophagectomy: A Multicenter Analysis of Total Minimally Invasive Esophagectomy in Low Risk Patients. Ann Surg 2017;266:814-21.

23. Hao Z, Zhenya S, Lei W. Esophageal-gastric anastomosis in radical resection of esophageal cancer under thoracoscopy combined with laparoscopy. J Coll Physicians Surg Pak 2014;24:754-6.

24. Gao S, An ZY. Control study of the short-term efficacy of Ivor-Lewis and McKeown operation by thoracoscopy and laparoscopy in esophageal squamous cell carcinoma. Chinese Journal of the Frontiers of Medical Science (Electronic Version) 2016;8:57-60.

25. Ling F. Short-term effects of full-endoscopic Ivor-lews Vs Mckeown esophagectomy on treatment of thoracic middle-lower segment esophageal carcinoma. Shandong Med J 2018;58:45-47.

26. Zhang L, Chen M, Zhang Y. The effect of minimally invasive esophagectomy Mckeown and Ivor-Lewis on pulmonary function in patients with esophageal carcinoma. Oncology Progress 2018;16:1604-7.

27. Ren W. Influence of Lvor-Lewis and McKeown surgery For middle and lower esophageal carcinoma in immune pain response, digestive function and prognosis. Pract J Clin Med 2018;15:246-8.

28. Chang T, Hsiao PN, Tsai MY, et al. Perioperative management and outcomes of minimally invasive 
esophagectomy: case study of a high-volume tertiary center in Taiwan. J Thorac Dis 2018;10:1670-6.

29. Zhai C. A comparison of short-term outcomes and quality of life between Ivor-Lewis and McKeown minimally invasive esophagectomy. Shandong University, 2016.

30. Mei S. Short-term efficacy of purse sting forceps IvorLewis approach and McKeown approach in minially invasive esophagectomy for middle-lower section esophageal cancer. Anhui Medical University, 2016.

Cite this article as: Wang $\mathrm{J}$, Hu J, Zhu D, Wang K, Gao C, Shan T, Yang Y. McKeown or Ivor Lewis minimally invasive esophagectomy: a systematic review and meta-analysis. Transl Cancer Res 2020;9(3):1518-1527. doi: 10.21037/tcr.2020.01.45
31. Inokuchi M, Kojima K, Kato K, et al. Risk factors for postoperative pulmonary complications after gastrectomy for gastric cancer. Surg Infect (Larchmt) 2014;15:314-21.

32. Scholtemeijer MG, Seesing MFJ, Brenkman HJF, et al. Recurrent laryngeal nerve injury after esophagectomy for esophageal cancer: incidence, management, and impact on short- and long-term outcomes. J Thorac Dis 2017;9:S868-78. 
PubMed and Medline search strategy

\#1, "Esophageal Neoplasms"[Mesh]

\#2, Esophageal Neoplasm

\#3, Neoplasm, Esophageal

\#4, Esophagus Neoplasm

\#5, Esophagus Neoplasms

\#6, Neoplasm, Esophagus

\#7, Neoplasms, Esophagus

\#8, Neoplasms, Esophageal

\#9, "Cancer of Esophagus"

\#10, "Cancer of the Esophagus"

\#11, Esophagus Cancer

\#12, Cancer, Esophagus

\#13, Cancers, Esophagus

\#14, Esophagus Cancers

\#15, Esophageal Cancer

\#16, Cancer, Esophageal

\#17, Cancers, Esophageal

\#18, Esophageal Cancers

\#19, "Esophagectomy"[Mesh]

\#20, Esophagectomies

\#21, Oesophagectomy

\#22, Oesophagectomies

\#23, \#1 OR \#2 OR \#3 OR \#4 OR \#5 OR \#6 OR \#7 OR \#8

OR \#9 OR \#10 OR \#11 OR \#12 OR \#13 OR \#14 OR \#15

OR \#16 OR \#17 OR \#18 OR \#19 OR \#20 OR \#21 OR \#22

\#24, "Video-Assisted Surgery"[Mesh]

\#25, Surgeries, Video-Assisted

\#26, Video Assisted Surgery

\#27, Video-Assisted Surgeries

\#28, Surgery, Video-Assisted

\#29, Surgery, Video Assisted

\#30, "Thoracic Surgery, Video-Assisted"[Mesh]

\#31, Surgeries, Video-Assisted Thoracic

\#32, Surgery, Video-Assisted Thoracic

\#33, Thoracic Surgeries, Video-Assisted

\#34, Thoracic Surgery, Video Assisted

\#35, Video-Assisted Thoracic Surgeries

\#36, Video-Assisted Thoracoscopic Surgery

\#37, Surgeries, Video-Assisted Thoracoscopic

\#38, Surgery, Video-Assisted Thoracoscopic

\#39, Thoracoscopic Surgeries, Video-Assisted

\#40, Thoracoscopic Surgery, Video-Assisted

\#41, Video Assisted Thoracoscopic Surgery

\#42, Video-Assisted Thoracoscopic Surgeries
\#43, Video-Assisted Thoracic Surgery

\#44, Video Assisted Thoracic Surgery

\#45, Surgery, Thoracic, Video-Assisted

\#46, VATS

\#47, VATSs

\#48, "Minimally Invasive Surgical Procedures"[Mesh]

\#49, Surgical Procedures, Minimal

\#50, Surgical Procedures, Minimal Access

\#51, Surgical Procedures, Minimally Invasive

\#52, Minimal Access Surgical Procedures

\#53, Minimal Surgical Procedure

\#54, Procedures, Minimally Invasive Surgical

\#55, Minimally Invasive Surgery

\#56, Surgeries, Minimally Invasive

\#57, Surgery, Minimally Invasive

\#58, Procedure, Minimal Surgical

\#59, Procedures, Minimal Access Surgical

\#60, Procedures, Minimal Surgical

\#61, Surgical Procedure, Minimal

\#62, Minimal Surgical Procedures

\#63, \#24 OR \#25 OR \#26 OR \#27 OR \#28 OR \#29 OR \#30

OR \#31 OR \#32 OR \#33 OR \#34 OR \#35 OR \#36 OR \#37

OR \#38 OR \#39 OR \#40 OR \#41 OR \#42 OR \#43 OR \#44

OR \#45 OR \#46 OR \#47 OR \#48 OR \#49 OR \#50 OR \#51

OR \#52 OR \#53 OR \#54 OR \#55 OR \#56 OR \#57 OR \#58

OR \#59 OR \#60 OR \#61 OR \#62

\#64, \#23 AND \#63

\#65, \#64 OR McKeown OR Ivor Lewis

\#66, "Cohort Studies"[Mesh]

\#67, Cohort Study

\#68, Studies, Cohort

\#69, Study, Cohort

\#70, Concurrent Studies

\#71, Studies, Concurrent

\#72, Concurrent Study

\#73, Study, Concurrent

\#74, Closed Cohort Studies

\#75, Cohort Studies, Closed

\#76, Closed Cohort Study

\#77, Cohort Study, Closed

\#78, Study, Closed Cohort

\#79, Studies, Closed Cohort

\#80, Analysis, Cohort

\#81, Cohort Analysis

\#82, Analyses, Cohort 
\#83, Cohort Analyses

\#84, Historical Cohort Studies

\#85, Cohort Study, Historical

\#86, Historical Cohort Study

\#87, Study, Historical Cohort

\#88, Incidence Studies

\#89, Incidence Study

\#90, Studies, Incidence

\#91, Study, Incidence

\#92, \#66 OR \#67 OR \#68 OR \#69 OR \#70 OR \#71 OR \#72

OR \#73 OR \#74 OR \#75 OR \#76 OR \#77 OR \#78 OR \#79

OR \#80 OR \#81 OR \#82 OR \#83 OR \#84 OR \#85 OR \#86

OR \#87 OR \#88 OR \#89 OR \#90 OR \#91

\#93, \#65 AND \#92

\section{Embase search strategy}

\#1, 'esophagus tumor'/exp

\#2, 'esophageal neoplasms'

\#3, 'esophageal tumor'

\#4, 'esophagus tumor'

\#5, 'esophagus tumour'

\#6, 'oesophageal neoplasms'

\#7, 'oesophageal tumor'

\#8, 'oesophageal tumour'

\#9, 'oesophagus tumor'

\#10, 'oesophagus tumour'

\#11, 'tumor, esophagus'

\#12, 'tumour, esophagus'

\#13, \#1 OR \#2 OR \#3 OR \#4 OR \#5 OR \#6 OR \#7 OR \#8

OR \#9 OR \#10 OR \#11 OR \#12

\#14, 'endoscopic surgery'/exp

$\# 15$, 'endoscopic resection'

\#16, 'endoscopic surgery'

\#17, 'resection, endoscopic'

$\# 18$, 'surgery, endoscopic'

\#19, 'surgery, video assisted'

\#20, 'surgical procedures, endoscopic'

\#21, 'video-assisted surgery'

\#22, 'video assisted thoracoscopic surgery'/exp

\#23, 'vats'

\#24, 'thoracic surgery, video-assisted'

\#25, 'video assisted thoracic surgery'
\#26, 'video assisted thoracoscopic surgery'

\#27, 'videothoracoscopic surgery'

\#28, 'minimally invasive surgery'/exp

\#29, 'mini-invasive surgery'

\#30, 'mini-invasive surgical procedure'

\#31, 'mini-invasive surgical procedures'

\#32, 'minimally invasive surgery'

\#33, 'minimally invasive surgical method'

\#34, 'minimally invasive surgical methods'

$\# 35$, 'minimally invasive surgical procedure'

\#36, 'minimally invasive surgical procedures'

\#37, 'minimally invasive surgical technique'

\#38, 'minimally invasive surgical techniques'

\#39, 'surgery, minimally invasive'

\#40, 'surgical procedures, minimally invasive'

\#41, \#14 OR \#15 OR \#16 OR \#17 OR \#18 OR \#19 OR \#20

OR \#21 OR \#22 OR \#23 OR \#24 OR \#25 OR \#26 OR \#27

OR \#28 OR \#29 OR \#30 OR \#31 OR \#32 OR \#33 OR \#34

OR \#35 OR \#36 OR \#37 OR \#38 OR \#39 OR \#40

\#42, \#13 AND \#41

\#43, \#42 OR 'McKeown' OR 'ivor lewis'

\#44, 'cohort analysis'/exp

\#45, 'analysis, cohort'

\#46, 'cohort analysis'

\#47, 'cohort fertility'

\#48, 'cohort life cycle'

\#49, 'cohort studies'

\#50, 'cohort study'

\#51, 'fertility, cohort'

\#52, \#44 OR \#45 OR \#46 OR \#47 OR \#48 OR \#49 OR \#50

OR \#51

\#53, \#43 AND \#52

\section{The Cochrane Library search strategy}

\#1, MeSH descriptor: [Esophageal Neoplasms] explode all trees

\#2, MeSH descriptor: [Esophagectomy] explode all trees

\#3, Oesophagectomy

\#4, Oesophagectomies

\#5, \#1 OR \#2 OR \#3 OR \#4

\#6, MeSH descriptor: [Video-Assisted Surgery] explode all trees 
\#7, MeSH descriptor: [Thoracic Surgery, Video-Assisted] explode all trees

\#8, MeSH descriptor: [Minimally Invasive Surgical

Procedures] explode all trees

\#9, \#6 OR \#7 OR \#8

\#10, \#5 AND \#9

\#11, \#10 OR McKeown OR Ivor Lewis
Wanfang Databases, China National Knowledge Infrastructure (CNKI) and Chinese VIP

Information search strategy

$\# 1$, McKeown

\#2, Ivor Lewis

\#3, Ivor-Lewis

\#4, \#1 OR \#2 OR \#3 
Table S1 The characteristics and quality assessment scores of included studies

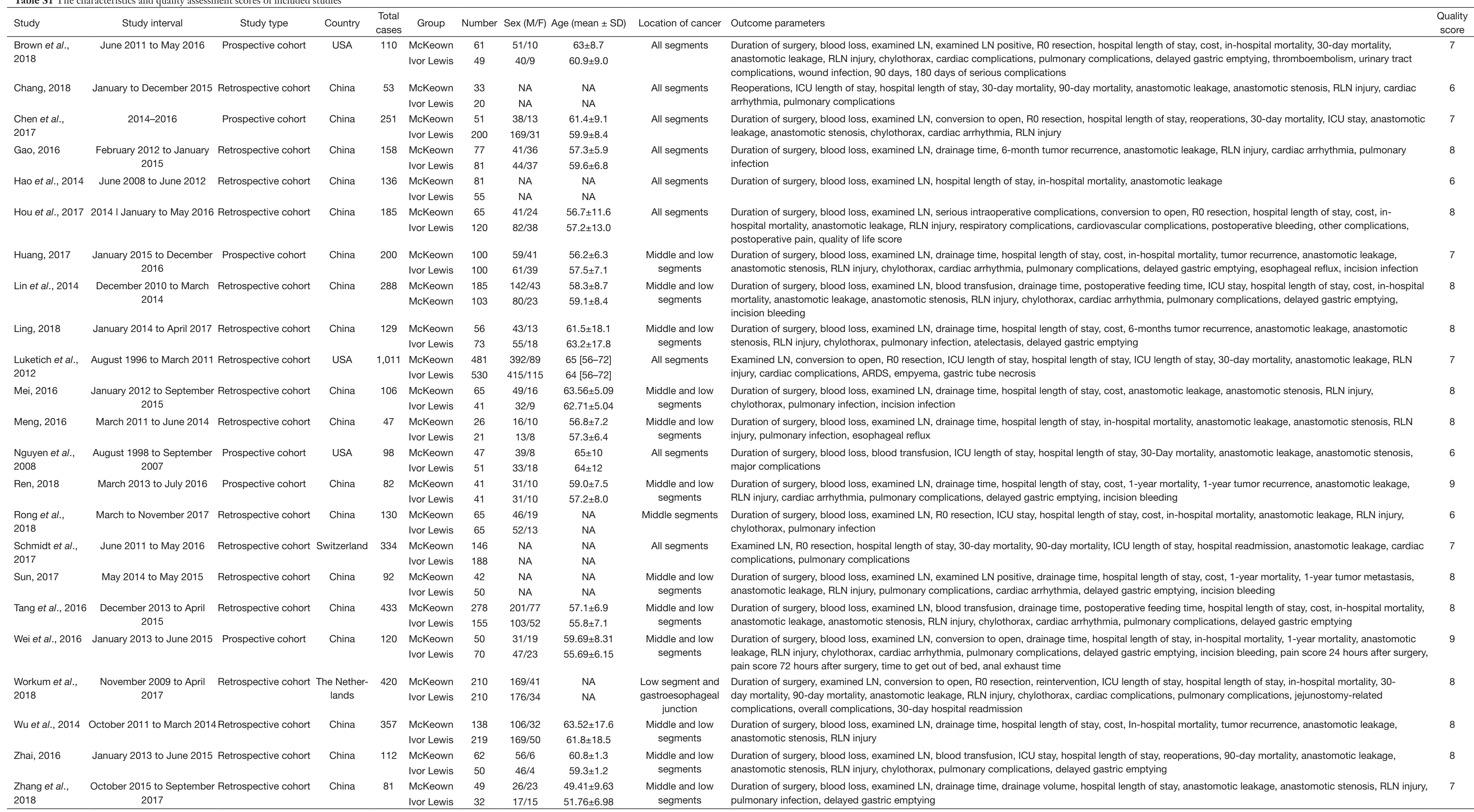

$\begin{array}{llllll} & 2017 & & \end{array}$ 